\title{
Social norms and attitude to diet and health in a sample of UK adults
}

\author{
L. Alahmari, C. Buckton, M. Martiniuc, A. Parrett and E. Combet \\ School of Medicine, Dentistry \& Nursing, Human Nutrition, New Lister Building, Glasgow Royal Infirmary, \\ Glasgow, G31 2ER
}

Food choices influence nutritional balance and energy intake, which are linked to the risk of developing obesity, CVD, and diabetes ${ }^{(1)}$. Social norms (SN; what society considers appropriate and acceptable conduct) are associated with social judgement and thus can influence eating behavior and food choice ${ }^{(1)}$. This study aims to investigate the impact of SN on attitudes towards food and diet in a sample of the UK adult population.

A survey, designed around the Theory of Planned Behaviour (TPB), was disseminated electronically and face-to-face to UK residents aged 18 years and above. Participants answered a questionnaire consisting of eighty-four questions using a mixture of Likert scales, true / false / not sure questions and open questions. Questions, constructed around seven dimensions based on the TPB, assessed: attitudes towards behavior (ATB), social norms (SN), perceived behavioural control (PBC), nutrition knowledge (NK), intention to change diet (ICD), current health status and demographic measures. SN were studied through 13 questions focusing on the beliefs / perceptions of family members, friends, doctors, culture, official resources, and media sources. The questionnaire Cronbach's alpha for the four sections ATB, SN, PBC and NK ranged between 0.375 and 0.77 . Multiple linear regression analyses were used to determine whether ATB, SN, PBC, NK, adjusted for gender and age, predicted the participants' intentions to change diet (measured in three specific questions). The data were analysed using SPSS software, version 21.0 for Windows (SPSS Inc., Chicago, IL, USA).

Participants $(\mathrm{n}=646)$ were mostly female $(70 \%)$ with median age 29 (IQR22). Statements with a strong agreement included 'I am interested in reading articles about food and health' (64\%), and 'I was brought up to always finish the food on my plate and still do' $(61 \%)$ while half agreed with 'I always check nutritional information labels before I buy any food $(49 \%)$ '. Half of the participants $(\mathrm{n}=373,58 \%)$ had 'already made a change to their diet',13\% had 'no plan to change their diet', and $56 \%$ intended to change their diet (further or start to make a change). ATB regarding diet and health was the strongest predictor of participants' intention to change their diet $(\mathrm{B}=0.141, \mathrm{P}=0.00)$, followed by $\mathrm{SN}(\mathrm{B}=0.134, \mathrm{P}=0.001)$. Interestingly, $\mathrm{PBC}(\mathrm{B}=0.009, \mathrm{P}=0.5)$ and $\mathrm{NK}$ $(\mathrm{B}=0.065, \mathrm{p}=0.095)$ did not influence ICD.

Our findings highlight attitudes and social norms as two important dimensions that interventions aiming to improve health through diet should tackle.

1. Wing RR and Hill JO (2001) Annual review of nutrition 21(1), 323-341. 